\title{
Authors' reply to Wang et al. comment on "Parkinson's disease associated with myasthenia gravis and rheumatoid arthritis"
}

\author{
Panagiotis Zis · Socrates J. Tzartos • \\ Antonios Tavernarakis
}

Received: 25 September 2014/ Accepted: 3 October 2014/Published online: 9 October 2014

(C) Springer-Verlag Italia 2014

To the Editor,

We would like to thank Wang et al. [1] for their comments on our case report [2]. They highlight the fact that patients presenting with parkinsonism may fell into either idiopathic Parkinson's disease (PD) or syndromes of secondary parkinsonism such as drug-induced parkinsonism, vascular parkinsonism, parkinsonism concomitant with autoimmune diseases, etc. [1]. In their comment, Wang et al. [1] dispute the diagnosis of idiopathic PD in our patient, since we haven't provided some clinical, imaging, and laboratory data.

Therefore, we would like to clarify that the diagnosis of idiopathic PD in our case was made 5 years prior to diagnosis of myasthenia gravis and rheumatoid arthritis. Apart from the initial clinical presentation of unilateral tremor, rigidity, and slowness of movement, the patient had had a brain MRI that was normal, and a DAT scan which was abnormal. Moreover, the patient's symptoms had a very good response on levodopa. Five years after the diagnosis of PD, the patient was admitted because of progressive weakness in the flexion of his neck. During his admission, which lead to the diagnosis of myasthenia gravis and rheumatoid arthritis and details of which have been published in our case report [2], he had been screened for autoimmune disorders. The following antibodies were

P. Zis $(\bowtie) \cdot$ A. Tavernarakis

Department of Neurology, Evangelismos General Hospital,

45-47 Ipsilantou Str., 10676 Athens, Greece

e-mail: takiszis@gmail.com

\section{S. J. Tzartos}

Hellenic Pasteur Institute, Athens, Greece

S. J. Tzartos

Tzartos Neurodiagnostics, Athens, Greece all negative; anti-nuclear antibodies (ANA), anti-doublestranded DNA (anti-dsDNA), perinuclear anti-neutrophil cytoplasmic antibodies (P-ANCA), cytoplasmic anti-neutrophil cytoplasmic antibodies (C-ANCA), anti-cardiolipin (ACLG and ACLM), anti-thyroid peroxidase (anti-TPO), anti-thyroglobulin (anti-TG). Moreover, his thyroid hormones titers (T3, T4, and TSH) were within normal limits. Finally, we must mention that the following paraneoplastic antibodies were also negative; $\mathrm{Hu}$ (ANNA-1), Ri (ANNA2), Yo (PCA-1), PNMA2 (Ma2/Ta), CV2 (CRMP-5), amphiphysin, recoverin, SOX1 (AGNA), zic4, and $\mathrm{Tr}$ (DNER).

In conclusion, we agree with Wang et al. that it is always important to be careful before diagnosing idiopathic PD, especially when causes of secondary parkinsonism have not been excluded. As mentioned above, with this letter, we would like to clarify that our patient had a diagnosis of idiopathic PD, which was diagnosed 5 years before the diagnosis of rheumatoid arthritis and myasthenia gravis.

\section{References}

1. Wang J, Wu X, Wu W, Zhang H, Liu K (2014) Parkinson's disease associated with myasthenia gravis and rheumatoid arthritis. Neurol Sci (Epub ahead of print)

2. Zis P, Argiriadou V, Temperikidis PP, Zikou L, Tzartos SJ, Tavernarakis A (2014) Parkinson's disease associated with myasthenia gravis and rheumatoid arthritis. Neurol Sci 35(5):797-799 